\title{
A COMPETITION GAME WITH KNOWLEDGE ACCUMULATION AND SPILLOVERS
}

\author{
GIAN-ITALO BISCHI* \\ Istituto di Scienze Economiche, Università di Urbino "Carlo Bo" \\ via Saffi 42, I-61029 Urbino, Italy \\ bischi@econ.uniurb.it \\ FABIO LAMANTIA \\ Dipartimento O.A.A.P., Facoltà di Economia, Università della Calabria \\ Via Pietro Bucci 3B-3C, I 87036, Arcavacata di Rende (CS), Italy \\ lamantia@unical.it
}

\begin{abstract}
In this paper a repeated game is proposed to model competition among firms, with profit maximizing resource allocation. The proposed model differs from the usual competition models because efforts that players exert are not seen as sunk costs, but they accumulate to form a stock of knowledge that has a cost-reducing effect. In modelling knowledge accumulation, we also consider the (knowledge) spillovers, that is, involuntary leakage of useful technological information. The game with $n$ boundedly rational agents is modelled by a $2 n$-dimensional discrete dynamical system, whose state variables are the $R \& D$ efforts and the stock of accumulated knowledge of each firm involved in the competition. The model is characterized by some counteracting forces: Efforts are costly but can increase future profits; immediate expenditures of each firm can have cost-reducing effects in the long run, since accumulated knowledge can decrease both own costs and competitors' ones, because of spillover effects. In the case of two homogeneous firms we prove the existence of a unique equilibrium and its stability. Starting from these analytic results, numerical simulations are performed in order to study the effects induced by heterogeneities between the players on stability and transient dynamics, as well as the influence of the main parameters on the basins of attraction.
\end{abstract}

Keywords: Competition games; $R \& D ;$ spillovers; dynamical systems; stability; oscillations.

Subject Classification: 91A, 91B, 70K, 70K20.

\section{Introduction}

Among the applications of mathematical modelling in economics, the description of marketing and industrial competition has a prominent place. In this setting, firms engage in profit maximizing resource allocation, and competitive (i.e., strategic) effects arise because the effectiveness of each firm's efforts is related to the efforts 
of its rivals. Typical situations described within this framework include brand competition for market share [see e.g., Monahan (1987), Hibbert and Wilkinson (1994), Cooper and Nakanishi (1988), Bischi et al. (2000)]. Stylized models of profit maximizing competitive resource allocation are often expressed in terms of effort $x_{i}$ that firm $i, i=1, \ldots, n$, decides to exert. As result of this efforts, in fact, firm $i$ is able to obtain a revenue that is proportional to its own effort $x_{i}$ and inversely proportional to the total efforts of all the competing firms. Formally, the profit of firm $i$ can be expressed as

$$
\pi_{i}=B \frac{a_{i} x_{i}}{\sum_{j=1}^{n} a_{j} x_{j}}-C_{i}(\cdot)
$$

where $B$ denotes the (fixed) sales potential of the market, the term $s_{i}=$ $a_{i} x_{i} / \sum a_{j} x_{j}$ represents the market share ${ }^{\mathrm{a}}$ of brand $i$, with weights $a_{i}>0$ whose values depend on the units used to measure efforts, ${ }^{\mathrm{b}}$ and $C_{i}$ is the cost associated with effort $x_{i}$.

When firms compete for the production of high tech goods, their efforts are mainly devoted to gain knowledge, intended in a broader meaning, ranging from basic notions to information about new technologies and skills in their adoption and employment. In many situations, such efforts can be identified with expenditures in Research and Development $(R \& D)$ activities. Indeed, a firm's optimal resource allocation in $R \& D$ is often necessary for introducing innovations before rivals or obtaining patents, with consequent competitive advantages or market shares greater than rivals. This kind of competition has been denoted as " $R \& D$ with rivalry" [e.g., Reinganum $(1981,1982)]$.

As stressed by many authors, when dealing with this kind of exertion some peculiar effects should be considered, such as the "knowledge capital stock" created by firms, that is, their gradually accumulated knowledge as a result of past efforts [see e.g., Nelson (1982), Confessore and Mancuso (2002)]. In addition, knowledge may spill from one firm to its competitors, due to the difficulty of protecting intellectual properties (Spence (1984), D'Aspremont and Jaquemin (1988), Bischi and Lamantia (2002), just to cite a few).

In this paper we propose a discrete-time competition game taking into account these issues, i.e., we model competition with profit maximizing effort decisions occurring over time, together with an associated process of knowledge accumulation and spillover effects. We assume that efforts are not "sunk costs", as they can be, at least partially, "capitalized" by each agent in the form of knowledge stock that tends to depreciate itself as time goes on, according to the obsolescence rate of information and technology.

For its particular set-up, the model could be employed to describe the dynamics of industrial sectors, such as the drug or the software industry, characterized by

af none of the competitors expend any effort, i.e., $x_{i}=0$ for each $i$, we just simply define $s_{i}=1 / n \forall i$.

${ }^{\mathrm{b}}$ Many authors set $a_{i}=1$ for each $i$, without loss of generality. 
high $R \& D$ costs and consequently by many inventions and patents. Consider, for instance, the drug industry where $R \& D$ costs are considerable, often outweighing the production costs altogether and consequently inventions are protected by patents. In this framework, $R \& D$ efforts could be measured in terms of scientific staff or expenditures in research activities, the stock of knowledge for a drug producer could be measured in terms of patents owned and its depreciation could be measured in terms of expiring patents.

Furthermore in our model the knowledge stock has a "cost reducing" effect, a quite common assumption in the literature [see e.g., Petit et al. (2000), Spence (1984)]. Another important feature of the proposed model is connected with the private and public nature of information and the related asymmetries of players in taking advantage from competitors' knowledge and/or in protecting their private knowledge. Following the idea of our previous example the causes of this leakage of knowledge could range from former employees who are hired by rival firms to industrial espionage. All in all the model is characterized by some counteracting forces: as usual, we have that efforts are costly but they can increase future profits; besides, indirect effects exist, since immediate costs can be recovered in the long run through the cost reducing effects of accumulated knowledge, and they can also decrease competitors' costs because of spillover effects.

The model is expressed as a repeated competition game, that takes the form of a discrete dynamical system, whose dynamic variables are efforts and knowledge accumulated by each competitor. In fact, the mechanism of knowledge accumulation with spillover effects is represented as a recursive process, so that its time evolution is represented by the repeated application of a map. In order to study the dynamic properties of the model, such as existence and stability of equilibria or more complex attractors, as well as questions related to transient dynamics and role of initial conditions, we shall consider the case of two competitors and the associated fourdimensional discrete dynamical system. For this system, we first consider the case of identical (or homogeneous) competitors, whose symmetry properties allow us to get complete analytic results on existence, stability and localization of the unique steady state. This benchmark case constitutes an useful guide for the numerical exploration of the effects induced by heterogeneities, and allows us to compare the cases of homogeneous and heterogeneous competitors. For example, heterogeneities may introduce oscillations in the long run behavior of the system, whereas oscillatory dynamics are completely ruled out with homogeneous players.

However we do not consider, at least in an explicit way, actual production of goods, which are sold in an oligopolistic market, but competition takes place in an abstract setting. In this way we can derive a model that is still tractable and represents a first step toward a full understanding of a more complete model.

The paper is organized as follows. In Sec. 2 we introduce the model, describing both the mechanism of knowledge accumulation and its embedding in an abstract model of competition. In Sec. 3 we study the dynamic properties of the duopoly, first under the assumption of homogeneous players, then, mainly by numerical methods, 
investigating the effects induced by the presence of heterogeneities. In Sec. 4 we describe the main economic interpretations of the results. Section 5 concludes.

\section{The Set-Up}

\subsection{A dynamic representation of accumulated knowledge with spillovers}

In this section we first propose a general model for the accumulation of knowledge over time, which can be expressed by a recursive formula, then we embed it in an abstract competition game.

Assume that there are $n$ firms, indexed by $i=1, \ldots, n$. Let $x_{i}(t)$ be a measure of the effort that firm $i$, at time period $t$, decides to make in order to increase its knowledge. The knowledge gain for firm $i$ during time period $t$ is assumed to be [see e.g., Spence (1984)]

$$
X_{i}(t)=x_{i}(t)+\sum_{j \neq i} \vartheta_{i j} x_{j}(t)
$$

where the parameters $\vartheta_{i j} \in[0,1]$ are intended to capture knowledge spillovers from firm $j$ to firm $i$ (intra-industry spillovers). If $\vartheta_{i j}=1$, then knowledge gained by firm $j$, via its own $R \& D$ efforts, is fully shared by firm $i$ (the case of $\vartheta_{i j}=1$ for each $i \neq j$ corresponds to the assumption of public domain knowledge). At the other limiting situation, $\vartheta_{i j}=0$, no knowledge from firm $j$ is transferred to firm $i$ (the case of $\vartheta_{i j}=0$ for each $i \neq j$ corresponds to the assumption of private companies that have proprietary rights to avoid any knowledge diffusion among competitors).

This is an extension of the model of knowledge gain proposed by Spence (1984). In fact, Spence assumes the same spillover parameter for all firms, i.e., $\vartheta_{i j}=\vartheta$ for each $i \neq j$, as a parameter that characterizes the whole industrial system, whereas our formulation allows one to consider heterogeneity (i.e., asymmetries) among the firms with respect to spillovers. In other words, we may consider situations where some firms are more efficient than others in spilling knowledge for free from competitors, or where some firms may be more efficient than others in avoiding the diffusion of the knowledge they gained as a result of their own efforts.

As argued above, we also want to consider that knowledge accumulates over time. This means that, at each time period, the knowledge of firm $i$ is not only increased by the last period knowledge gain $X_{i}(t)$, but it also benefits of the (discounted) knowledge gains of the past. To model this, we represent the knowledge accumulated by firm $i$, up to period $t$, by

$$
z_{i}(t)=\sum_{k=0}^{t} \rho^{t-k} X_{i}(k)
$$


where the parameter $\rho \in[0,1]$ represents a discount factor which "exponentially discounts older information". This captures the idea that the knowledge accumulated by firm $i, z_{i}(t)$, includes not only the current gain $X_{i}(t)$, but also the knowledge gained by past efforts. However, the stock of knowledge may depreciate as well, as outlined by several authors [see e.g., Nelson (1982), Confessore and Mancuso (2002)]. The value of $\rho$ gives a measure of how rapidly information becomes obsolete: values close to 1 represent a system where even the results of very old efforts are important at time $t$, whereas values close to 0 imply that only very recent efforts give significant contributions to the present knowledge.

Starting from (3), the knowledge capital stock of each firm $i$ at time period $t$ can be obtained recursively (i.e., inductively) as

$$
z_{i}(t)=X_{i}(t)+\rho \sum_{k=0}^{t-1} \rho^{t-1-k} X(k)=x_{i}(t)+\sum_{j \neq i} \vartheta_{i j} x_{j}(t)+\rho z_{i}(t-1)
$$

This recursive formula can be interpreted as follows the accumulated knowledge at time $t$ is the sum of the knowledge $X_{i}(t)$, acquired during last round, and a discounted fraction of the knowledge capital stock of the previous period $(t-1)$.

\subsection{The competition game with cost externalities}

We consider an abstract model (i.e., without explicitly considering actual production) where $n$ firms, labelled by $i, i=1, \ldots, n$, at each time period $t$ choose their $R \& D$ expenditures in order to maximize their expected next period profit

$$
\pi_{i}^{e}(t+1)=\frac{a_{i} x_{i}(t+1)}{a_{i} x_{i}(t+1)+\sum_{j \neq i} a_{j} x_{j}^{e}(t+1)}-C_{i}^{t+1}
$$

where $x_{j}^{e}(t+1)$ represents the effort that firm $i$ expects that the competing firm $j$ will exert at time $(t+1)$, and $C_{i}^{t+1}$ represents the cost at time $t+1$. Our key assumption is that the cost at time $t+1$ for player $i, C_{i}^{t+1}$, is a (differentiable) function of both the current efforts and the knowledge accumulated up to period $t$, i.e., $C_{i}^{t+1}=C_{i}^{t+1}\left(x_{i}(t+1), z_{i}(t)\right)$, with (see e.g., Spence, 1984; Petit and Tolwinski, 1999)

$$
\frac{\partial C_{i}^{t+1}}{\partial x_{i}(t+1)} \geq 0 ; \quad \frac{\partial C_{i}^{t+1}}{\partial z_{i}(t)} \leq 0
$$

These assumptions capture the idea that $R \& D$ efforts are an investment with an immediate negative effect, given by the cost of current effort $x_{i}$, and a delayed beneficial one given by more knowledge, which enhances the skillfulness and makes technology advance. This last fact can be restated by saying that more knowledge 
implies that $R \& D$ results are obtained more efficiently, i.e., with lower unitary costs. $^{\text {c }}$ Moreover it is natural to assume that [see Spencer and Brander (1983)]

$$
\frac{\partial^{2} C_{i}^{t+1}}{\partial x_{i}(t+1) \partial z_{i}(t)}<0
$$

i.e., increasing the stock of knowledge lowers the marginal cost of the $R \& D$ effort.

A simple functional form that satisfies these assumptions can be obtained by assuming that $C_{i}^{t+1}$ is linear in the current efforts $x_{i}(t+1)$ and inversely proportional to $z_{i}(t)$, i.e., knowledge accumulated up to period $t$,

$$
C_{i}^{t+1}\left(x_{i}(t+1), z_{i}(t)\right)=\frac{\alpha_{i} x_{i}(t+1)+\beta_{i}}{\lambda_{i} z_{i}(t)+\zeta_{i}}
$$

However, for sake of simplicity, in the following we shall consider a particular form for the cost function, that still maintains the above described features [see Petit et al. (2000) for a similar specification], given by

$$
C_{i}^{t+1}\left(x_{i}(t+1), z_{i}(t)\right)=\frac{x_{i}(t+1)}{1+\lambda_{i} z_{i}(t)}
$$

where the parameters $\lambda_{i} \geq 0, i=1, \ldots, n$, can be interpreted as a measure of efficiency in the use of the knowledge stock (and the related technology) to decrease costs for further innovation.

With cost function (6) the expected profit (5) becomes

$$
\pi_{i}^{e}(t+1)=\frac{a_{i} x_{i}(t+1)}{a_{i} x_{i}(t+1)+\sum_{j \neq i} a_{j} x_{j}^{e}(t+1)}-\frac{x_{i}(t+1)}{1+\lambda_{i} z_{i}(t)}
$$

where, without loss of generality, we set $B=1$, i.e., a unitary market size. The expected share per unit effort is $a_{i} /\left(a_{i} x_{i}+\sum_{j \neq i} a_{j} x_{j}^{e}\right)$, whereas the expected profit margin per unit investment is

$$
\frac{\pi_{i}^{e}}{x_{i}}=\frac{a_{i}}{a_{i} x_{i}+\sum_{j \neq i} a_{j} x_{j}^{e}}-\frac{1}{1+\lambda_{i} z_{i}}
$$

At each time period $t$, every firm $i$ decides the effort for the next period, $x_{i}(t+1) \geq 0$, by maximizing the expected net profit $\pi_{i}^{e}(t+1)$, i.e., $x_{i}(t+1)=$ $\operatorname{argmax}_{x_{i}(t+1)} \pi_{i}^{e}(t+1), i=1, \ldots, n$. Assuming interior optimum, simple

${ }^{\mathrm{c}}$ This is a common sense statement: An agent that already has a stock of accumulated knowledge, because she/he already performed a given job in the past, will obtain the same results at a lower cost with respect to competitors that have no background knowledge. 
differentiation gives

$$
\frac{\partial \pi_{i}^{e}(t+1)}{\partial x_{i}(t+1)}=\frac{a_{i} \sum_{j \neq i} a_{j} x_{j}^{e}(t+1)}{\left[a_{i} x_{i}(t+1)+\sum_{j \neq i} a_{j} x_{j}^{e}(t+1)\right]^{2}}-\frac{1}{1+\lambda_{i} z_{i}(t)}=0 \quad i=1, \ldots, n
$$

at the optimum. This gives a quadratic equation for $x_{i}(t+1)$, from which the optimum is easily obtained as a function of the expected efforts of the competitors $x_{j}^{e}(t+1), j \neq i$, and the accumulated knowledge $z_{i}(t)$ :

$$
x_{i}(t+1)=\frac{1}{a_{i}}\left(-\sum_{j \neq i} a_{j} x_{j}^{e}(t+1)+\sqrt{a_{i}\left(1+\lambda_{i} z_{i}(t)\right) \sum_{j \neq i} a_{j} x_{j}^{e}(t+1)}\right)
$$

Notice that, according to $(2)$ and $(3), z_{i}(t)$, the knowledge accumulated by firm $i$ up to period $t$, depends on past efforts both of firm $i$ itself and of its competitors, because of the presence of spillover effects. So, besides the interdependence caused by the term $a_{i} x_{i} /\left(a_{i} x_{i}+\sum_{j \neq i} a_{j} x_{j}^{e}\right)$, as in standard competition models, another source of interdependence is present, due to the positive cost externalities induced by accumulated knowledge with spillover effects. In fact, while in a standard competition game (i.e., $\lambda_{i}=0, i=1, \ldots, n$ ) effort $x_{i}$ represents a sunk cost for firm $i$, according to Eq. (6) effort is a retrievable investment in the sense that it increases the knowledge stock thus allowing future costs reduction.

Notice also that, as the solution of the optimization problem for firm $i$ depends on $x_{j}^{e}(t+1)$, an assumption on how firm $i$ forms expectations about competitors' choices is necessary in order to close the model. One may assume that $x_{j}^{e}(t+1)=$ $x_{j}(t+1)$, i.e., every firm is able to know in advance the competitors' decisions (perfect foresight assumption). However, this is often considered a too demanding assumption, and a lower degree of information is assumed by using some adaptive learning scheme such that $x_{j}^{e}(t+1)$ is computed on the basis of the values observed in the past. In the following, for sake of simplicity, we shall assume naive expectations (or expectations á la Cournot), i.e., $x_{j}^{e}(t+1)=x_{j}(t)$. This can be interpreted by saying that, due to the lack of information about competitors' choices, each player guesses that in the next time period the competitors will exert the same effort as in the current period. Of course, this is a quite trivial assumption, even if it is very common in the literature on dynamic oligopoly games [since Cournot (1838), see also Okuguchi (1976), Puu and Sushko (2002)], on dynamic brand competition models [e.g., Bischi et al. (2000), Bischi and Kopel (2003)], on dynamic rent-seeking games [e.g., Okuguchi and Szidarovszky (1999), Xu and Szidarovszky (1999)].

In order to investigate the effects, on the existence and stability of the equilibria, of the parameters of the model, as well as the kind of convergence (monotonic or oscillatory) and the dynamic behavior obtained when no stable steady states exist, in the following we shall restrict our analysis to the case of only two competing firms $(n=2)$, i.e., a duopoly dynamic game. We consider Eq. (9) with naive expectations 
and $a_{1}=a_{2}=a$, together with the recursive relations (4) which describe the time evolution of knowledge accumulated by each firm, and we get a discrete dynamical system in the four dynamic variables $\left(x_{1}, x_{2}, z_{1}, z_{2}\right) \in \mathbb{R}^{4}$

$$
\left\{\begin{aligned}
x_{1}(t+1)= & -x_{2}(t)+\sqrt{x_{2}(t)\left(1+\lambda_{1} z_{1}(t)\right)} \\
x_{2}(t+1)= & -x_{1}(t)+\sqrt{x_{1}(t)\left(1+\lambda_{2} z_{2}(t)\right)} \\
z_{1}(t+1)= & -x_{2}(t)+\sqrt{x_{2}(t)\left(1+\lambda_{1} z_{1}(t)\right)} \\
& +\vartheta_{12}\left(-x_{1}(t)+\sqrt{x_{1}(t)\left(1+\lambda_{2} z_{2}(t)\right)}\right)+\rho z_{1}(t) \\
z_{2}(t+1)= & -x_{1}(t)+\sqrt{x_{1}(t)\left(1+\lambda_{2} z_{2}(t)\right)} \\
& +\vartheta_{21}\left(-x_{2}(t)+\sqrt{x_{2}(t)\left(1+\lambda_{1} z_{1}(t)\right)}\right)+\rho z_{2}(t)
\end{aligned}\right.
$$

with initial conditions that, according to the definition (3) of the variables $z_{i}$, are given by

$$
x_{1}(0) ; \quad x_{2}(0) ; \quad z_{1}(0)=x_{1}(0)+\vartheta_{12} x_{2}(0) ; \quad z_{2}(0)=x_{2}(0)+\vartheta_{21} x_{1}(0)
$$

i.e., the initial conditions of the four-dimensional dynamical system (10) must be taken in a two-dimensional submanifold (a plane) of $\mathbb{R}^{4}$ in order to obtain trajectories of the process we are modelling. This means that if each firm selects an initial level of effort $x_{i}(0), i=1,2$, then the sequence of successive efforts is obtained inductively by iterating (10) starting from initial conditions (11). It is plain that only non negative values of the dynamical variables $x_{i}$ are economically meaningful. Moreover, the dynamical system (10) is defined only for non negative values of $x_{i}$, $i=1,2$. So, starting from a given initial condition (11), a feasible time evolution of the system is obtained only if the sequence $\left(x_{1}(t), x_{2}(t)\right), t=0,1,2, \ldots$ along a trajectory of $(10)$ is entirely contained in the non negative orthant of $\mathbb{R}^{4}$. Accordingly, we shall call feasible trajectory a trajectory with the property stated above, and feasible set the set of points $x_{1}(0), x_{2}(0)$ such that the corresponding initial conditions (11) generate feasible trajectories.

\section{Local and Global Analysis}

As usual in the study of nonlinear dynamical systems, the first analysis step is the localization of the fixed points (or steady states) and the analysis of their stability. The fixed points of (10) are the solutions of the algebraic system obtained from (10) with $x_{i}(t+1)=x_{i}(t)$ and $z_{i}(t+1)=z_{i}(t), i=1,2$. Of course, we are only interested in the fixed points with positive coordinates. After some simple algebraic manipulations, the equations which give the equilibrium values of the two efforts become

$$
\left\{\begin{array}{l}
x_{1}^{2}+\left(1-\frac{\lambda_{1} \vartheta_{12}}{1-\rho}\right) x_{2}^{2}+\left(2-\frac{\lambda_{1}}{1-\rho}\right) x_{1} x_{2}-x_{2}=0 \\
\left(1-\frac{\lambda_{2} \vartheta_{21}}{1-\rho}\right) x_{1}^{2}+x_{2}^{2}+\left(2-\frac{\lambda_{2}}{1-\rho}\right) x_{1} x_{2}-x_{1}=0
\end{array}\right.
$$


and the corresponding equilibrium values for the knowledge capital stocks are given by $z_{1}=\left(x_{1}+\vartheta_{12} x_{2}\right) /(1-\rho)$ and $z_{2}=\left(x_{2}+\vartheta_{21} x_{1}\right) /(1-\rho)$. Equations (12) represent two conic-sections (ellipses, parabolas or hyperboles, according to the parameters) whose intersections in the positive orthant of the $\left(x_{1}, x_{2}\right)$ plane correspond to the equilibrium points of the game. In general, a study of existence, uniqueness and stability of the equilibria of the model (10) is not an easy task. However, a particular case with a complete analytic characterization is obtained by assuming homogenous firms, in the sense that they are characterized by identical values of the parameters

$$
\lambda_{1}=\lambda_{2}=\lambda ; \quad \vartheta_{12}=\vartheta_{21}=\vartheta .
$$

This symmetric situation can be considered as a benchmark case, since the analytical results that we shall obtain under assumption (13) will constitute an useful starting point for the study of the discrete dynamical system (10) with heterogeneities in knowledge spillovers, i.e., $\vartheta_{12} \neq \vartheta_{21}$ and/or in cost reducing parameters, i.e., $\lambda_{1} \neq \lambda_{2}$.

\subsection{Existence and stability of the steady state with homogeneous firms}

Under assumption (13) the system (12) is symmetric, and the analysis of existence and stability of equilibria can be carried out analytically. In fact, it is straightforward to see that a unique positive solution of (12) exists, given by

$$
\begin{aligned}
E=\left(x^{*}, x^{*}, z^{*}, z^{*}\right) \quad \text { with } \quad x^{*} & =\frac{1-\rho}{4(1-\rho)-\lambda(1+\vartheta)} \text { and } \\
z^{*} & =\frac{1+\vartheta}{4(1-\rho)-\lambda(1+\vartheta)}
\end{aligned}
$$

provided that

$$
\lambda(1+\vartheta)<4(1-\rho) .
$$

This condition states that an equilibrium exists only if the parameters $\lambda, \vartheta$ and $\rho$ are sufficiently small, i.e., the accumulated knowledge does not cause a too strong reduction of unitary cost and past knowledge does not accumulate too much. Notice that condition (15) is never satisfied if $\lambda(1+\vartheta)>4$, and if $\lambda(1+\vartheta)<4$ then it is satisfied for sufficiently small values of the parameter $\rho$, i.e., $\rho<1-\lambda(1+\vartheta) / 4$. This is quite intuitive, because the process we are describing can have a steady state, characterized by constant values of $x_{i}$ and $z_{i}$, only if the rate at which new knowledge is gained equals the rate at which old knowledge is dissipated, so that stationariety is impossible without sufficiently high knowledge dissipation.

In order to study the stability properties of the equilibrium $E$, we must evaluate the eigenvalues of the Jacobian matrix of the map (10) computed at the equilibrium (see Appendix). Under assumption (13) of homogeneous players, the computation of the eigenvalues gives $\eta_{1}=0 ; \eta_{2}=0 ; \eta_{3}=0.25(1+\vartheta) \lambda+\rho ; \eta_{4}=0.25(1-\vartheta) \lambda+\rho$. 
It is easy to see that if $E$ exists, i.e., condition (15) holds, then $0 \leq \eta_{i}<1$ for each $i=1, \ldots, 4$. So, the following proposition can be stated (see Appendix):

Proposition 1. If (13) and (15) hold, then the unique equilibrium (14) exists and is asymptotically stable.

Condition (15) clearly shows that the existence of $E$ is favored by decreasing values of $\lambda, \vartheta$ and $\rho$, and if the existence condition (15) is satisfied, then the symmetric equilibrium value $x_{1}^{*}=x_{2}^{*}=x^{*}(\rho, \lambda, \vartheta)$ given in (14) is an increasing function of the parameters $\rho, \lambda$ and $\vartheta$, i.e., if one or more of these parameters are increased, then at the equilibrium the firms will exert more effort. Moreover, smaller values of $\lambda$ and $\rho$ increase the speed of convergence to the stable equilibrium, since both $\eta_{3}$ and $\eta_{4}$ decrease if $\lambda$ and $\rho$ are decreased. In this sense, it may be said that decreasing values of the parameters $\lambda$ and $\rho$ reinforce the stability of the equilibrium $E$. Notice also that the convergence is nonoscillatory around $E$, because the eigenvalues are real and non negative.

However, Proposition 1 only concerns local asymptotic stability of the unique positive equilibrium in the case of homogeneous firms, where local, as usual, is referred to trajectories that start from initial conditions sufficiently close to $E$. Indeed, convergence cannot be obtained for every initial condition, i.e., $E$ cannot be globally stable, because if $x_{i}(0)$ is too high then from (10) one gets a negative $x_{j}(1)$, that is, an unfeasible trajectory is obtained. Moreover, if (15) is not satisfied, then no positive steady state exists, and all the initial conditions generate unfeasible trajectories.

It is interesting to compute numerically the extension of the set of initial conditions that generate feasible trajectories converging to $E$, and how it is influenced by the parameters of the model. For example, in Fig. 1(a), obtained with parameters $\lambda=0.2, \vartheta=0.2$ and $\rho=0.5$, the white region represents the set of points that generate feasible trajectories converging to $E$, whereas the grey region represents the unfeasible set. The projection ${ }^{\mathrm{d}}$ in the $\left(x_{1}, x_{2}\right)$ plane of a typical trajectory that converges to $E$ is shown in the Fig. 1(a), starting from initial condition $\left(x_{1}(0), x_{2}(0)\right)=(0.1,1)$, denoted by "i.c." in the figure. The convergence to $E$ appears to be very fast. Indeed, for the set of parameters used to obtain Fig. 1(a), the nonvanishing eigenvalues are $\eta_{3}=0.56$ and $\eta_{4}=0.54$, so the dominant eigenvalue $^{\mathrm{e}}$ is 0.56 . The situation shown in Fig. 1(a) can be compared with the one in Fig. 1(b), obtained with the same $\lambda$ and $\rho$ and with a greater value of the spillover parameter $\vartheta$, namely $\vartheta=0.8$. In this case, the dominant eigenvalue is $\eta_{3}=0.59$, i.e., we have a slightly slower convergence, but we can notice that the basin of

\footnotetext{
${ }^{d}$ The trajectories of the dynamical system (10) evolve in the four dimensional space $\mathbb{R}^{4}$, so the figures we propose represent projections on the two-dimensional subspace $\left(x_{1}, x_{2}\right)$ where the time evolution of efforts is represented.

e The dominant eigenvalue is defined as the eigenvalue with greatest modulus. If its modulus, say $|z|$, is less than one, a small displacement from the equilibrium reduces at a rate proportional to $|z|^{t}$, i.e., $1 /|z|$ gives an estimate of the speed of convergence.
} 


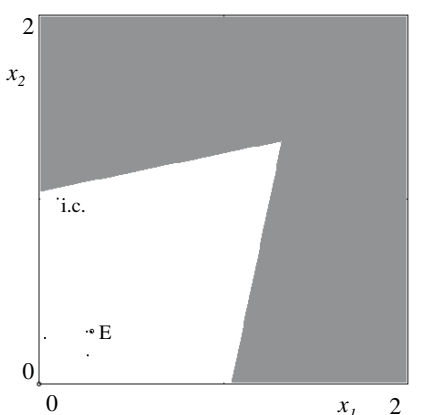

(a)

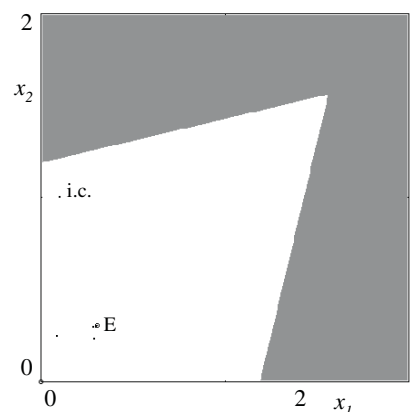

(b)

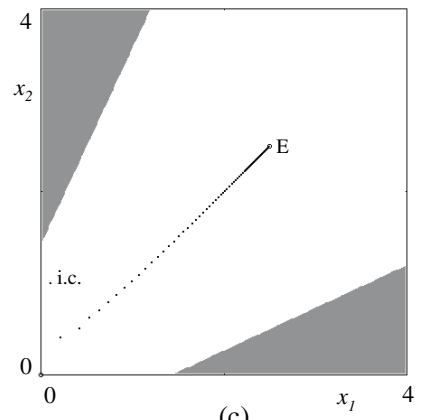

(c)

Fig. 1. Duopoly model with homogeneous firms. The white region represents the set of points that generate feasible trajectories converging to $E$, the grey region represent the unfeasible set. (a) $\lambda=0.2, \vartheta=0.2$ and $\rho=0.5$. The equilibrium $E$ is represented together with the projection in the $\left(x_{1}, x_{2}\right)$ plane of the trajectory starting from initial condition "i.c." $\left(x_{1}(0), x_{2}(0)\right)=(0.1,1)$. (b) Parameters $\lambda$ and $\rho$ are the same as in (a) and $\vartheta=0.8$. (c) Parameters $\vartheta$ and $\rho$ are the same as in (a) and $\lambda=1.5$.

attraction of $E$ has a larger extension. This suggests that an increase of the overall (homogeneous) spillover parameter may give two contrasting effects about the so called "relative stability" of the equilibrium $E$ : a weaker stability, in the sense of a slower convergence, and a stronger stability, in the sense of a greater basin of attraction [on this point, see the definitions and the discussion in Bischi and Marimon (2001)]. This twofold effect is even more evident if, starting from the set of parameters of Fig. 1(a), we increase the parameter $\lambda$. This is shown in Fig. 1(c), obtained with parameters $\lambda=1.5, \vartheta=0.2$ and $\rho=0.5$. In this case, the set of points that generate feasible trajectories converging to $E$ (white region) is greatly enlarged, and the dominant eigenvalue is close to one, being $\eta_{3}=0.95$, so that the convergence to $E$ is very slow. This is confirmed by the plot, shown in Fig. 1(c), of the trajectory that starts from the same initial condition $\left(x_{1}(0), x_{2}(0)\right)=(0.1,1)$ as in Figs. 1(a) and 1(b). It is evident that in this case the convergence requires much more iterations. This is confirmed by many other numerical explorations, from which we can conjecture that even if decreasing values of $\lambda$ favors the existence of $E$ and also the speed of convergence, an opposite effect is obtained on its robustness measured in terms of the extension of the basin. A similar effect is observed for the parameter $\vartheta$, whereas changes in the parameter $\rho$ seem to have no remarkable effects on the basin.

So, putting together analytic and numeric results, we can say that in the case of two homogeneous firms a unique locally stable equilibrium exists if the condition (15) holds, the convergence to it is always nonoscillatory (no overshooting), with increasing speed of convergence and smaller basin of attraction for decreasing values of the parameters $\lambda$ and $\vartheta$. However, as we shall see in the next section, quite different dynamic scenarios can be observed in the case of heterogeneous firms, that is, if the two firms are characterized by different parameter values. 


\subsection{Numerical simulations in the case of heterogeneous firms}

If assumption (13) of identical competitors is relaxed, the computation of the steady states requires numerical methods, because the analytic solution of the fourth degree algebraic system (12) does not give compact expressions in terms of elementary functions. The same holds for the computation of the eigenvalues of the Jacobian matrix (A.1). So, numerical simulations of the discrete dynamical system (10) are necessary in order to investigate the effects of heterogeneities, i.e., $\lambda_{1} \neq \lambda_{2}$ and/or $\vartheta_{12} \neq \vartheta_{21}$, on the dynamic behavior of the repeated competition game.

We now propose some sequences of numerical explorations, starting from the benchmark case of homogenous players, and then we introduce different kinds of heterogeneities between the players in order to study their effects on the long run dynamics of the system. For example, Fig. 2(a) is obtained with $\lambda_{1}=\lambda_{2}=0.2$, like in Fig. 1(a), $\rho=0.4$ and a strong heterogeneity in the spillover parameters, namely $\vartheta_{12}=0$ and $\vartheta_{21}=1$. This means that knowledge freely spills from firm 1 to firm 2, whereas no knowledge spillovers occur from firm 2 to firm 1 (i.e., firm 2 is able to protect its efforts). Two effects can be noticed from Fig. 2(a) first, the stable equilibrium $E=\left(x_{1 E}, x_{2 E}\right)$ is characterized by $x_{2 E}>x_{1 E}$, i.e., at the equilibrium firm 2 exerts more effort than firm 1; second, the convergence occurs through damped oscillations, i.e., overshooting effects occur before convergence [see the projection of the trajectory shown in Fig. 2(a), starting from the initial condition $\left.\left(x_{1}(0), x_{2}(0)\right)=(0.2,0.3)\right]$. Similar effects are obtained by assuming identical spillover parameters and different values of cost-reducing parameters,

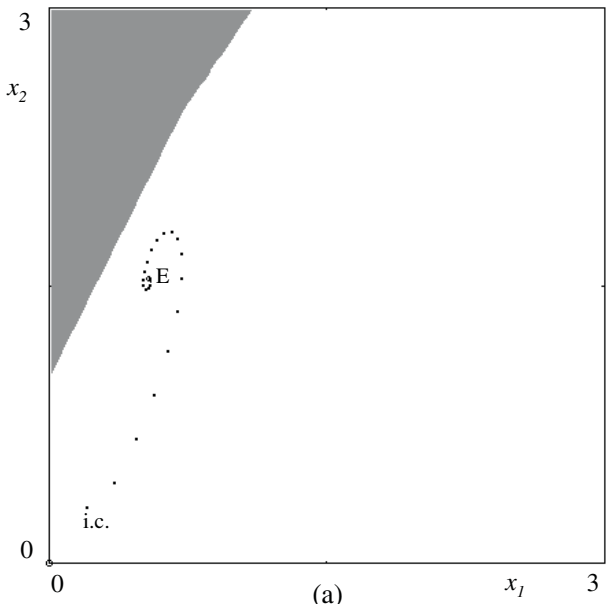

(a)

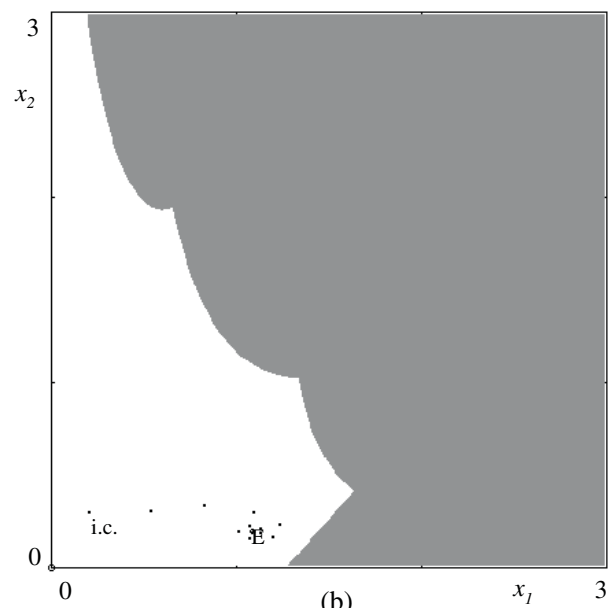

(b)

Fig. 2. Duopoly model with heterogeneous firms. The white region represents the set of points that generate feasible trajectories converging to $E$, the grey region represents the unfeasible set. (a) $\lambda_{1}=\lambda_{2}=0.2, \rho=0.4, \vartheta_{12}=0$ and $\vartheta_{21}=1$. The stable equilibrium $E$ is represented together with the projection in the $\left(x_{1}, x_{2}\right)$ plane of the trajectory starting from initial condition "i.c." $\left(x_{1}(0), x_{2}(0)\right)=(0.2,0.3)$ (b) The same as (a) with $\vartheta_{12}=\vartheta_{21}=0.3, \lambda_{1}=4.5, \lambda_{2}=0.7$. 
i.e., $\lambda_{1} \neq \lambda_{2}$. For example, Fig. 2(b) is obtained with the same value of $\rho$ as Fig. 2(a), $\vartheta_{12}=\vartheta_{21}=0.3, \lambda_{1}=4.5, \lambda_{2}=0.7$. Also in this case the convergence to $E$ is oscillatory, and $x_{1 E}>x_{2 E}$, i.e., the firms that experiences more evident cost reducing effects of efforts invests more. In Fig. 2(b) another consequence of the introduction of differences between the parameters $\lambda_{1}$ and $\lambda_{2}$ can be noticed, related to the shape of the boundary that separates the feasible set from the unfeasible set.

More evident oscillations are obtained by introducing differences both between the parameters $\lambda_{1}$ and $\lambda_{2}$ and between the spillover parameters $\vartheta_{12}$ and $\vartheta_{21}$. As heterogeneities are strengthened, the oscillations become more and more evident (the imaginary part of two complex conjugate eigenvalues increases) until the equilibrium loses stability via a Neimark-Hopf bifurcation (i.e., a pair of complex conjugate eigenvalues exits the unit circle of the complex plane) and a stable closed invariant curve is created around the unstable equilibrium along which quasi-periodic self sustained oscillations take place. This situation is shown in Fig. 3, obtained with $\rho=0.3, \lambda_{1}=4.5, \lambda_{2}=0.7, \vartheta_{12}=0.8, \vartheta_{21}=0.1$. As expected, the equilibrium $E$ is characterized by $x_{1 E}>x_{2 E}$, because in this case firm 1 is more capable to protect its efforts results (being $\vartheta_{12}>\vartheta_{21}$ ) and the cost reducing effects are stronger for firm 1 than for firm 2 (being $\lambda_{1}>\lambda_{2}$ ). However, the equilibrium $E$ is unstable, and the attractor to which the generic feasible trajectory converges is represented by a stable closed orbit around it. The trajectory starting from the initial condition $\left(x_{1}(0), x_{2}(0)\right)=(0.2,0.3)$ is represented in Fig. 3, both projected in the $\left(x_{1}, x_{2}\right)$

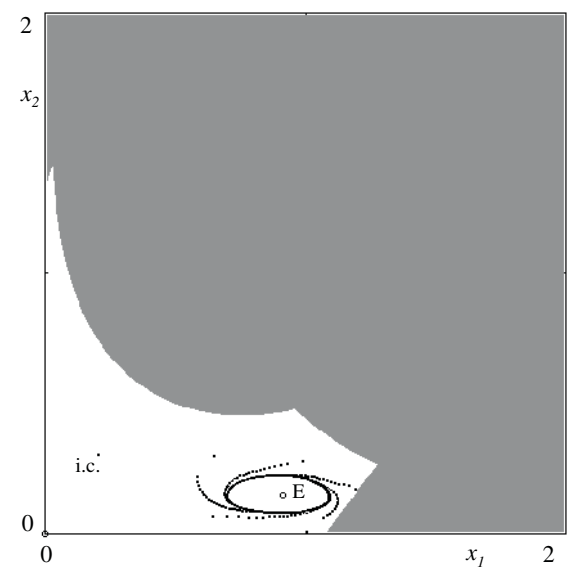

(a)
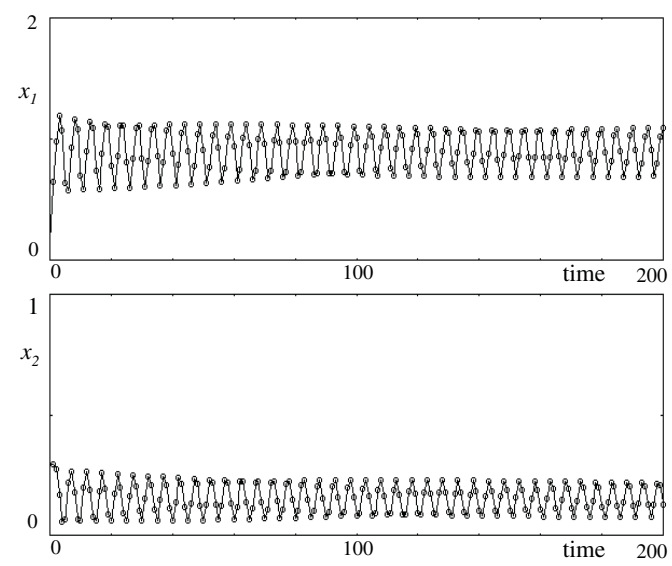

(b)

Fig. 3. Duopoly model with heterogeneous firms, with parameters $\rho=0.3, \lambda_{1}=4.5, \lambda_{2}=0.7$, $\vartheta_{12}=0.8, \vartheta_{21}=0.1$. The white region represents the set of points that generate feasible trajectories converging to a stable closed invariant orbit around the unstable equilibrium $E$, the grey region represents the unfeasible set. (a) Projection in the $\left(x_{1}, x_{2}\right)$ plane of the trajectory starting from initial condition "i.c." $\left(x_{1}(0), x_{2}(0)\right)=(0.2,0.3)$ (b) The same trajectory is represented by showing the sequences $x_{1}(t)$ and $x_{2}(t)$ versus time. 


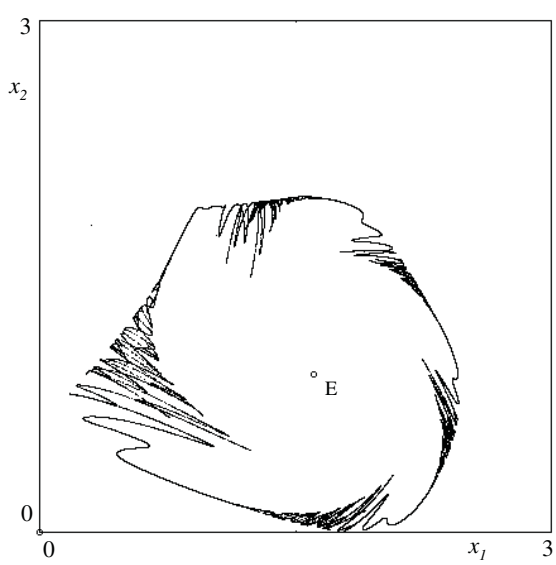

(a)
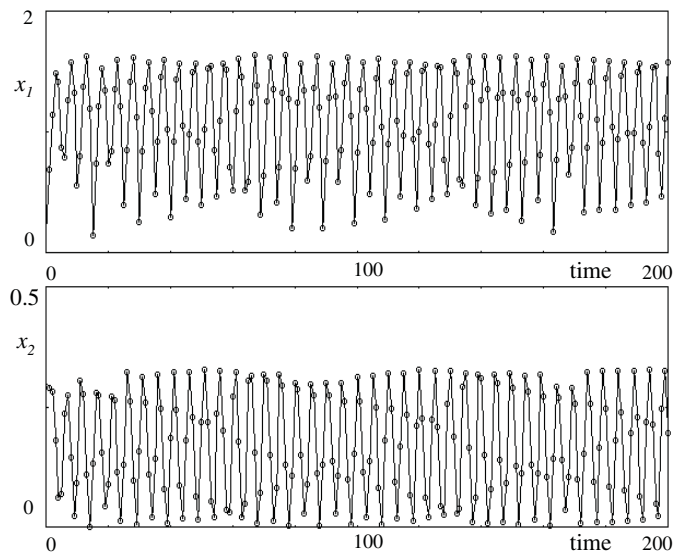

(b)

Fig. 4. Duopoly model with heterogeneous firms, with parameters $\rho=0.2825, \lambda_{1}=6.49$, $\lambda_{2}=0.5, \vartheta_{12}=\vartheta_{21}=0.5$. (a) Projection, in the $\left(x_{1}, x_{2}\right)$ plane, of the chaotic attractor to which the trajectories starting close to the unstable equilibrium $E$ converge (b) The same trajectory is represented by showing the sequences $x_{1}(t)$ and $x_{2}(t)$ versus time.

subspace [Fig. 3(a)] and by showing the sequences $x_{1}(t)$ and $x_{2}(t)$ versus time [Fig. 3(b)].

To conclude we note that even chaotic attractors can be observed by increasing the difference between the parameters $\lambda_{1}$ and $\lambda_{2}$, as shown in Fig. 4 where $\rho=0.2825, \lambda_{1}=6.49, \lambda_{2}=0.5, \vartheta_{12}=\vartheta_{21}=0.5$.

\section{Economic Interpretation of the Results}

In this section we describe some economic consequences arising from the study of the proposed model. As stressed in the introduction, there are counteracting forces at work in the model; for example direct effects of increasing efforts are the increment of (expected) market share and profits but also increasing values of current costs, whereas strategic long-run effects are related to the increment of the knowledge stock, which, in turn, induces a decrement in own (future) costs but also in rivals' ones through spillover effects. Hence it is important to study how these counteracting forces act in a dynamic framework.

We proved that in the case of homogeneous players, under suitable conditions on the parameters, a unique and locally stable steady state exists, and the convergence is always nonoscillatory (i.e., no overshooting effects can occur). The condition on the parameters that ensure existence and stability of the unique equilibrium in the case of homogeneous players, essentially requires sufficiently small values of the parameter $\rho$. This is quite intuitive within this context, since the process we are describing can have a steady state, characterized by constant values of $x_{i}$ and $z_{i}$, only if the rate at which new knowledge is gained equals the rate at which old 
knowledge is dissipated, so that stationariety is impossible without sufficiently high knowledge dissipation.

The fact that, for suitable values of the parameters, the repeated game has a locally stable equilibrium, can be described by saying that if the initial condition is not too far from the equilibrium then the game, repeatedly played by not fully rational players who have naive expectations about the competitors' decisions, will converge, in the long run, to the same equilibrium as in the one-shot game obtained under the assumption of full rationality. Moreover, in the case of homogeneous players, they will make identical choices in the long run.

The equilibrium values at the steady state $x_{i}^{*}(\rho, \lambda, \vartheta), i=1,2$, are increasing functions of the three parameters, so we can say that the capacity in making a better use of the knowledge stock (increments in $\lambda$ ) and/or the ability to exploit rivals' efforts (increments in $\vartheta$ ) induces an increment in efforts, hence in competitiveness between the firms. In addition, when knowledge spillovers are present, the increase of $\lambda, \vartheta$ or $\rho$ can lead to aggregate expenditures that are higher than the value of the rent, hence producing the effect known as "rent dissipation". However, at least in the homogeneous case, we proved that high parameters levels always imply the non-boundedness of the time evolution of the system, i.e., firms tend to increment their efforts indefinitely (divergent trajectories).

It is worthwhile to consider the role of the parameters not only for the existence and stability of the steady state, but also in the relative stability, by studying their influence on the speed of convergence and on the extension of the basin of attraction, since it is possible that these two aspects of relative stability lead to contrasting conclusions, i.e., a stronger stability in the sense of faster convergence may be associated with a weaker stability in the sense of basin's extension, and vice versa. This feature of the model has been proved by numerical analysis, hence showing that, in same cases (e.g., decreasing $\lambda$ and/or $\vartheta$ ), the nearer the model is to the perfect foresight case (fast convergence to the steady state), the nearer the initial condition should be to the equilibrium (small basin of attraction), otherwise divergent (i.e., not economic meaningful) trajectories are obtained. The fact that the steady state cannot be globally stable has an obvious economic explanation, because if the costs of a firm are higher than the unitary market size, then negative profits are expected and this will force the firm to stop efforts for the next period, and consequently it will exit the market, as our model assumes that $R \& D$ efforts are necessary for staying in the market.

In addition, numerical explorations of the model clearly show that the presence of heterogeneities has not only the expected effect of giving an asymmetric steady state, where the two players have different equilibrium efforts in the long run dynamics, but also induces oscillations during the transient before the long run dynamics settle down to the steady state.

The results obtained in the case of two heterogenous competitors show that the firm that is more efficient in avoiding spillovers and/or in exploiting its own knowledge to reduce costs, will invest more in the long run. Moreover, severe 
heterogeneities may destabilize the equilibrium and cause the creation of persistent self-sustained oscillations. This means that the players, due to heterogeneities and naive expectations about competitors' behavior, are not able to learn playing an equilibrium, and the repeated game continues to move around the equilibrium.

\section{Concluding Remarks}

In this paper we proposed a repeated discrete-time competition game, where we introduced a dynamic mechanism for the description of knowledge accumulation over time, as effect of $R \& D$ expenditures. The model also includes other effects, such as dissipation of knowledge and involuntary transmission of knowledge between firms (spillover effects).

In general there is not an obvious measuring unit for the knowledge stock, even if we may assume, for instance, that it can be measured in terms of patents owned, and its dissipation may be due to patents expiration. As an explanatory example we recalled that such a situation can be observed in the drug industry or in the competition among software houses, where $R \& D$ costs often outweigh the production costs altogether, and spillover effects are important.

By properly tuning the spillover parameters $\vartheta_{i j}$, the model allows us to study the effects of different balances between public and private knowledge, including cases of asymmetric spillovers, and, by tuning the memory parameter $\rho$, we can simulate different degrees of knowledge depreciation. The introduction, as dynamic variables, of the levels of accumulated knowledge of the competing firms, increases the number of dimensions of the discrete time dynamical system, so, after a general $n$-firms formulation of the model, we restricted our analysis to the case of two competing firms, represented by a four-dimensional dynamical system. A complete analytic characterization of the conditions for existence, uniqueness and stability of the steady state is given with homogeneous players, whereas insights on the case with heterogeneous players as well as on the global dynamic of the model are mainly obtained through numerical methods.

A possible extension of the proposed model could include the concept of absorptive capacity, introduced by Cohen and Levinthal (1989). They argue that firm $i$, in order to be able to identify, assimilate, and exploit knowledge from its rival firms, has to be endowed with its own knowledge, which in turn enhances firm $i$ 's absorptive capacity. This effect can be easily introduced in our model by considering spillover parameters as functions of the accumulated knowledge, i.e., $\vartheta_{i j}=\vartheta_{i j}\left(z_{i}\right)$, instead of constant coefficients.

In this paper we referred to market share attraction models, because they have been frequently used in empirical work [see, e.g., Bultez and Naert (1975), Naert and Weverbergh (1981)], in theoretical research of economics, game theory, operations research and marketing [see, for example, Monahan and Sobel (1994), Monahan (1987), Schmalensee (1976), Case (1979, Ch. 4), Cooper and Nakanishi (1988)] and in economic dynamics as well [see e.g., Bischi et al. (2000), Kopel et al. (2000)]. 
However, our results can be easily applied to rent seeking games. In fact, the same expression (1) of the profit function is also used in the framework of rent-seeking games [see e.g., Pérez-Castrillo and Verdier (1993), Nitzan (1994), Okuguchi and Szidarovszky (1999), Bischi et al., (2001)] where $B$ represents the total rent and $s_{i}$ is the probability to win the rent.

We also observe that the abstract and stylized model of this paper could be employed to study particular competition models with accumulation effects. For example, the iterative mechanism of knowledge accumulation could be usefully applied to the oligopoly model with market saturation described in Okuguchi and Szidarovszky (1999, Ch. 4), where an accumulation effect of goods that customers buy is considered, through a recursive process which is very similar to the knowledge accumulation mechanism of our model. So, the model proposed in this paper can be seen as an abstract benchmark from which further studies and applications can be developed.

\section{Acknowledgments}

The authors thank Giorgio Calcagnini, Herbert Dawid, Michael Kopel and an anonymous referee for their many valuable suggestions and remarks. We also acknowledge comments from participants at the 7th WHEIA, Trieste 2002, at 2nd MDEF, Urbino 2002 and 2nd Conference on Dynamic Oligopolies, Odense 2003. The usual disclaimer applies. This work has been performed within the activity of the national research project "Nonlinear models in economics and finance: complex dynamics, disequilibrium, strategic interactions", MIUR, Italy, and under the Joint Research Grant (0382) "Reconsideration of economic dynamics from a new perspective of nonlinear theory", Chuo University, Japan.

\section{Appendix}

The study of the local stability requires the study of the eigenvalues of the Jacobian matrix of the map (10), given by

$$
D T=\left[\begin{array}{cccc}
0 & \frac{1}{4 A_{2}\left(x_{2}, z_{1}\right)}-1 & \lambda_{1} A_{2}\left(x_{2}, z_{1}\right) & 0 \\
\frac{1}{4 A_{1}\left(x_{1}, z_{2}\right)}-1 & 0 & 0 & \lambda_{2} A_{1}\left(x_{1}, z_{2}\right) \\
\vartheta_{12}\left(\frac{1}{4 A_{1}\left(x_{1}, z_{2}\right)}-1\right) & \frac{1}{4 A_{2}\left(x_{2}, z_{1}\right)}-1 & \lambda_{1} A_{2}\left(x_{2}, z_{1}\right)+\rho & \lambda_{2} \vartheta_{12} A_{1}\left(x_{1}, z_{2}\right) \\
\frac{1}{4 A_{1}\left(x_{1}, z_{2}\right)}-1 & \vartheta_{21}\left(\frac{1}{4 A_{2}\left(x_{2}, z_{1}\right)}-1\right) & \lambda_{1} \vartheta_{21} A_{2}\left(x_{2}, z_{1}\right) & \lambda_{2} A_{1}\left(x_{1}, z_{2}\right)+\rho
\end{array}\right]
$$

where

$$
A_{1}\left(x_{1}, z_{2}\right)=\frac{x_{1}}{2 \sqrt{x_{1}\left(1+\lambda_{2} z_{2}\right)}} \quad \text { and } \quad A_{2}\left(x_{2}, z_{1}\right)=\frac{x_{2}}{2 \sqrt{x_{2}\left(1+\lambda_{1} z_{1}\right)}}
$$

computed at the fixed points. 
The Jacobian matrix in the homogeneous case is given by

$$
J=\left[\begin{array}{cccc}
0 & \frac{\sqrt{x_{2}\left(1+\lambda z_{1}\right)}}{2 x_{2}}-1 & \frac{\lambda x_{2}}{2 \sqrt{x_{2}\left(1+\lambda z_{1}\right)}} & 0 \\
\frac{\sqrt{x_{1}\left(1+\lambda z_{2}\right)}}{2 x_{1}}-1 & 0 & 0 & \frac{\lambda x_{1}}{2 \sqrt{x_{1}\left(1+\lambda z_{2}\right)}} \\
\vartheta\left(\frac{\sqrt{x_{1}\left(1+\lambda z_{2}\right)}}{2 x_{1}}-1\right) & \frac{\sqrt{x_{2}\left(1+\lambda z_{1}\right)}}{2 x_{2}}-1 & \frac{\lambda x_{2}}{2 \sqrt{x_{2}\left(1+\lambda z_{1}\right)}}+\rho & \frac{\lambda x_{1} \vartheta}{2 \sqrt{x_{1}\left(1+\lambda z_{2}\right)}} \\
\frac{\sqrt{x_{1}\left(1+\lambda z_{2}\right)}}{2 x_{1}}-1 & \vartheta\left(\frac{\sqrt{x_{2}\left(1+\lambda z_{1}\right)}}{2 x_{2}}-1\right) & \frac{\lambda x_{2} \vartheta}{2 \sqrt{x_{2}\left(1+\lambda z_{1}\right)}} & \frac{\lambda x_{1}}{2 \sqrt{x_{1}\left(1+\lambda z_{2}\right)}}+\rho
\end{array}\right]
$$

or

$$
J=\left[\begin{array}{cccc}
0 & \frac{1}{4 A_{2}}-1 & \lambda A_{2} & 0 \\
\frac{1}{4 A_{1}}-1 & 0 & 0 & \lambda A_{1} \\
\theta\left(\frac{1}{4 A_{1}}-1\right) & \frac{1}{4 A_{2}}-1 & \lambda A_{2}+\rho & \lambda \theta A_{1} \\
\frac{1}{4 A_{1}}-1 & \theta\left(\frac{1}{4 A_{2}}-1\right) & \lambda \theta A_{2} & \lambda A_{1}+\rho
\end{array}\right]
$$

being $A_{i}=\frac{x_{i}}{2 \sqrt{x_{i}\left(1+\lambda z_{j}\right)}}, i=1,2, i \neq j$.

In the origin $E_{1}$ the map is obviously not differentiable.

In the point $E_{2}$ the Jacobian reduces to

$$
J\left(E_{2}\right)=\left[\begin{array}{cccc}
0 & 0 & \frac{\lambda}{4} & 0 \\
0 & 0 & 0 & \frac{\lambda}{4} \\
0 & 0 & \rho+\frac{\lambda}{4} & \frac{\lambda \vartheta}{4} \\
0 & 0 & \frac{\lambda \vartheta}{4} & \rho+\frac{\lambda}{4}
\end{array}\right]
$$

and the eigenvalues are given $\eta_{1}=0 ; \eta_{2}=0 ; \eta_{3}=\frac{1}{4}(1+\vartheta) \lambda+\rho ; \eta_{4}=-\frac{1}{4}(\vartheta-1) \lambda+\rho$. The proof of Proposition 1 is then straightforward.

\section{References}

Bischi, G. I., Gardini, L. and Kopel, M. [2000] "Analysis of global bifurcations in a market share attraction model," Journal of Economic Dynamics and Control 24, 855-879.

Bischi, G. I. and Kopel, M. [2003] "Multistability and path dependence in a dynamic brand competition model," Chaos, Solitons $\&$ Fractals 18, 561-576.

Bischi, G. I., Kopel, M. and Naimzada, A. [2001] "On a rent-seeking game described by a non-invertible iterated map with denominator," Nonlinear Analysis, Theory, Methods and Applications 47, 5309-5324.

Bischi, G. I. and Marimon, R. [2001] "Global stability of inflation target policies with adaptive agents," Macroeconomic Dynamics 5, 148-179.

Bischi, G. I. and Lamantia, F. [2002] "Nonlinear duopoly games with positive cost externalities due to spillover effects," Chaos, Solitons $\mathcal{E}$ Fractals 13, 701-721.

Bultez, A. V. and Naert, P. A. [1975] "Consistent sum-constrained models," Journal of the American Statistical Association 70, 529-535.

Case, J. H. [1979] Economics and the Competitive Process (New York University Press). 
Cohen, W. M. and Levinthal, D. A. [1989] "Innovation and learning: The two faces of R\&D," The Economic Journal 99, 569-596.

Confessore, G. and Mancuso, P. [2002] "A dynamic model of R\&D competition," Research in Economics 56, 365-380.

Cooper, L. G. and Nakanishi, M. [1988] Market-Share Analysis (Kluwer Academic Publishers).

Cournot, A. [1838] Recherches sur les Principles Mathématiques de la Théorie des Richesses (Hachette, Paris).

D'Aspremont, C. and Jaquemin, A. [1988] "Cooperative and noncooperative R\&D in duopoly with spillovers," American Economic Review 78, 1133-1137.

Hibbert, B. and Wilkinson, I. F. [1994] "Chaos theory and the dynamics of marketing systems," Journal of the Academy of Marketing Science 22, 218-233.

Kopel, M., Bischi, G. I. and Gardini, L. [2000] "On new phenomena in dynamic promotional competition models with homogeneous and quasi-homogeneous firms," in Interaction and Market Structure. Essays on Heterogeneity in Economics, eds. Delli Gatti, D., Gallegati, M. and Kirman, A. P. (Springer-Verlag), pp. 57-87.

Monahan, G. E. [1987] "The Structure of Equilibria in market share attraction models," Management Science 33, 228-243.

Monahan, G. E. and Sobel, M. J. [1994] "Stochastic dynamic market share attraction games," Games and Economic Behavior 6, 130-149.

Naert, Ph. and Weverbergh, M. [1981] "On the predition power of market share attraction models," Journal of Marketing Research 18, 146-153.

Nelson, R. R. [1982] "The role of knowledge in R\&D efficiency," The Quarterly Journal of Economics 97(3), 453-470.

Nitzan, S. [1994] "Modelling rent-seeking contest," European Journal of Political Economy 10, 41-60.

Okuguchi, K. [1976] Expectations and Stability in Oligopoly Models (Springer-Verlag).

Okuguchi, K. and Szidarovszky, F. [1999] The Theory of Oligopoly with Multi-Product Firms 2nd edition (Springer).

Pérez-Castrillo, J. D. and Verdier, T. [1993] "A general analysis of rent-seeking games," Public Choice 73, 335-350.

Petit, M. L., Sanna-Randaccio, F. and Tolwinski, B. [2000] "Innovation and foreign investment in a dynamic oligopoly," International Game Theory Review 2(1), 1-28.

Petit, M. L. and Tolwinski, B. [1999] "R\&D cooperation or competition?" European Economic Review 43, 185-208.

Puu, T. and Sushko, I. (eds.) [2002] Oligopoly Dynamics: Models and Tools (SpringerVerlag).

Reinganum, J. F. [1981] "Dynamic games of innovation," Journal of Economic Theory $\mathbf{2 5}, 21-41$.

Reinganum, J. F. [1982] "A dynamic game for R and D: Patent protection and competitive behavior," Econometrica, 50, 671-688.

Schmalensee, R. [1976] "A model of promotional competition in oligopoly," Review of Economic Studies 43, 493-507.

Spence, M. [1984] "Cost reduction, competition, and industry performance," Econometrica 52, 101-121.

Spencer, B. J. and Brander, J. A. [1983] "International R\&D rivalry and Industrial strategy," The Review of Economic Studies 50(4), 707-722.

$\mathrm{Xu}$, L. and Szidarovszky, F. [1999] "The stability of dynamic rent-seeking games," International Game Theory Review 1, 87-102. 